\title{
Methicillin-resistant Staphylococcus aureus infection is associated with increased mortality
}

\section{Michael Otto}

Pathogen Molecular Genetics Section, Laboratory of Human Bacterial Pathogenesis, National Institute of Allergy \& Infectious Diseases, NIH, Bethesda, MD, USA =motto@niaid.nih.gov

Evaluation of: Hanberger $\mathrm{H}$, Walther S, Leone $\mathrm{M}$ et al. Increased mortality associated with meticillin-resistant Staphylococcus aureus (MRSA) infection in the intensive care unit: results from the EPIC II study. Int. J. Antimicrob. Agents $38(4), 331-335$ (2011). Methicillin resistance is a widespread and major source of treatment complication in Staphylococcus aureus infections. Whether infections with methicillin-resistant $S$, aureus are associated with a worse clinical outcome, such as higher mortality, has remained controversial. Analyzing data from a large, global multicenter study, Hanberger et al. demonstrate that methicillin-resistant S. aureus infections are associated with approximately $50 \%$ higher mortality in the intensive care unit and significantly more frequent among critically ill patients than infections with methicillin-susceptible $S$. aureus. These findings call for the implementation or continuation of active methicillin-resistant $S$. aureus surveillance measures.

Staphylococcus aureus is a major human pathogen, causing a multitude of moderately severe to serious and frequently fatal hospital-associated infections around the world [1]. Additionally, it is a premier source of community associated skin and soft tissue infections [2]. It is mostly known for causing infections in hospitalized, predisposed patients such as the elderly, immunocompromised or those undergoing surgery. Among those, infections of the respiratory tract, abscesses and bacteremia are particularly frequent and may develop into extremely dangerous and life-threatening diseases such as necrotizing pneumonia or necrotizing fasciitis. Treatment is often complicated by the fact that many $S$. aureus strains carry multiple antibiotic resistance determinants [3]. The most notorious are those conferring resistance to methicillin and other $\beta$-lactam antibiotics, known as methicillin-resistant $S$. aureus (MRSA). In many countries, most hospital associated $S$. aureus strains are resistant to methicillin, with MRSA rates exceeding 50\% [4].

Many hospitals and countries have initiated MRSA control measures to limit the spread of MRSA in hospitals and ascertain fast analysis of antibiotic resistance patterns in infecting strains to direct physicians to appropriate antibiotic therapy. Such measures are based on the assumption that cases of MRSA are clinically more complicated, in that they are associated with a worse clinical outcome than those caused by methicillin-susceptible $S$. aureus (MSSA). However, studies that have investigated the correlation between methicillin resistance in $S$. aureus and clinical outcome have reported conflicting results [5-8]. This may be owing to smaller sample sizes or the fact that often only one infection type was investigated, such as pneumonia [9]. Furthermore, one major reason underlying those discrepancies may be the pronounced global clonality of MRSA strains: usually, one or only a few MRSA clones are endemic in a given geographical region [10] Thus, results of a geographically limited study are naturally influenced by the properties of the MRSA clones present in that location, such as relative virulence or level of resistance to methicillin.

In the present study by Hanberger et al. [11], the authors evaluated a large cohort of patients $(13,796)$ from an extended and geographically diverse array of hospitals (1265 from 75 countries). Their analysis is based on the 1-day pointprevalence EPIC II study, which showed that approximately $51 \%$ of patients were infected, $20.5 \%$ of them with $S$. aureus, with an MRSA rate of approximately $50 \%$ [12]. The main goal of the Hanberger et al. study was to evaluate whether there are clinical characteristics distinguishing MRSA from MSSA infection.

The authors note that there were considerable geographical variations in MRSA rates when infections were grouped by continent of origin. MRSA rates were especially high in North America and Asia, which is in accordance with other studies. They were also high in Africa, but this may not be significant owing to the low
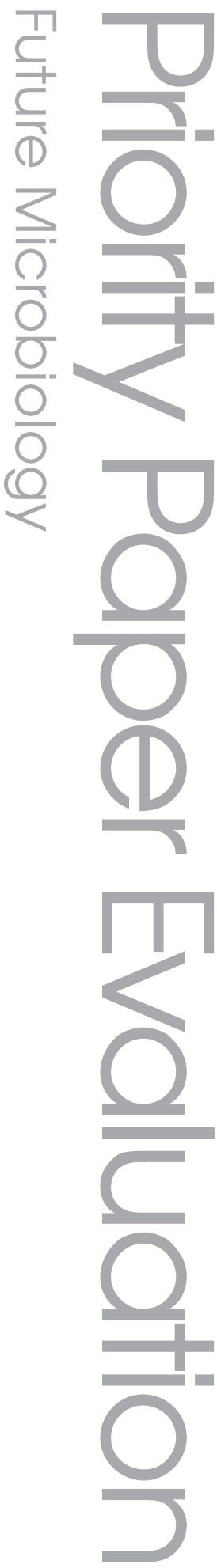

\section{Keywords}

- antibiotic resistance

- hospital-associated

infections $=$ MRSA

- Staphylococcus aureus

Future $\because$ Medicine part of 
number of cases from Africa that were investigated. They were quite low in western Europe; one may speculate whether this is owing to the rigid MRSA control measures in place at least in some European countries.

Not surprisingly, and in accordance with common observations, the main site of MRSA infection was the respiratory tract and MRSA patients were more likely to be admitted from the hospital ward than from the emergency department. Furthermore, antibiotic therapy on the study day was appropriate in $81.4 \%$ of cases for MRSA and $87.9 \%$ for MSSA, reflecting that an unrecognized methicillin resistance status leads to increased probability of inappropriate antibiotic therapy.

Importantly, it was found that mortality rates were significantly higher among MRSA than MSSA patients (in the intensive-care unit [ICU], 29.1 vs $20.5 \%$; $p<0.01$; in the hospital, 36.4 vs $27 \%$; $p<0.01$ ). Differences were even more pronounced in western Europe (in the ICU, 29.4 vs $16.7 \%$; $<<0.001$; in the hospital, 39.3 vs $22.8 \%$; $p<0.001$ ), while case numbers were too low to give significant results for other continents. Upon multivariate logistic regression analysis, MRSA was associated with a higher mortality risk in hospitals. Furthermore, in the ICU, MRSA patients more frequently had underlying comorbid conditions, such as cancer or chronic renal failure, compared with MSSA patients.

\section{Conclusion \& future perspective}

The clear strength of the Hanberger et al. study is its large sample size, giving reliable information on the correlation of methicillin resistance with increased mortality when analyzed on average in a large number of countries, despite its focus on western Europe. However, given that different MRSA clones with different characteristics dominate in specific geographical locations, the situation in such locations may differ. This may explain, in addition to the small sample sizes of many other studies, why results were divergent in more geographically limited studies and especially those not predominantly investigating the situation in western Europe.

The authors also stress that one limitation of their study was the lack of discrimination between community and healthcare-associated MRSA infections. In their study, MSSA dominated in patients admitted from the emergency department, but this situation may be different in countries that have reported a high rate of community-associated MRSA infections, such as the USA [2].

Most notably, the findings of the Hanberger $e t$ al. study underscore the need for testing of methicillin resistance upon hospital admission as well as stringent measures to control the spread of MRSA. It is a well-known fact that in countries that have introduced stringent MRSA control measures, such as The Netherlands, MRSA rates are exceptionally low [13]. Some countries with high MRSA rates have recently introduced such measures, for example France [14], and it will be of great interest to follow the development of MRSA infection rates in those locations.

Finally, it needs to be stressed that a conclusion such as "MRSA are associated with higher mortality rates than MSSA" is easily misunderstood as "MRSA are more virulent than MSSA". The latter is a purely microbiological assessment and not a conclusion to be drawn from this exclusively clinical study. The investigation of virulence factors underlying epidemic

Executive summary

\section{Objective}

- To assess whether there is a correlation between methicillin-resistant strains of Staphylococcus aureus and clinical outcome, such as increased mortality.

\section{Methods}

- Analysis of demographic, physiological, bacteriological and therapeutic data in a cohort of 13,796 adults from 1265 intensive-care units and 75 countries.

\section{Results}

- Methicillin-resistant S. aureus infections in the intensive-care unit are associated with a 50\% higher mortality risk than methicillinsusceptible S. aureus infections.

- Methicillin-resistant S. aureus infections are associated with underlying comorbid conditions more frequently than methicillin-susceptible $S$. aureus.

\section{Conclusion}

- Active surveillance for methicillin-resistant S. aureus and standard infection prevention measures are encouraged, particularly in critically ill patients. 
outbreaks of particular MRSA strains is an important task of current MRSA research that is needed to complement clinical studies such as the Hanberger et al. study.

\section{Financial \& competing interests disclosure}

This work was supported by the Intramural Research Program of the National Institute of Allergy and
Infectious Diseases, US NIH. The author has no other relevant affliations or financial involvement with any organization or entity with a financial interest in or financial conflict with the subject matter or materials discussed in the manuscript apart from those disclosed.

No writing assistance was utilized in the production of this manuscript.

\section{References}

Papers of special note have been highlighted as:

- of considerable interest

1. Lowy FD. Staphylococcus aureus infections. N. Engl. J. Med. 339(8), 520-532 (1998).

2. DeLeo FR, Otto M, Kreiswirth BN, Chambers HF. Community-associated methicillin-resistant Staphylococcus aureus. Lancet 375(9725), 1557-1568 (2010).

3. Lowy FD. Antimicrobial resistance: the example of Staphylococcus aureus. J. Clin. Invest. 111(9), 1265-1273 (2003).

4. Hiramatsu K, Cui L, Kuroda M, Ito T. The emergence and evolution of methicillinresistant Staphylococcus aureus. Trends Microbiol. 9(10), 486-493 (2001).

5. Blot SI, Vandewoude KH, Hoste EA, Colardyn FA. Outcome and attributable mortality in critically Ill patients with bacteremia involving methicillin-susceptible and methicillin-resistant Staphylococcus aureus. Arch. Intern. Med. 162(19), 2229-2235 (2002).

6. Cosgrove SE, Sakoulas G, Perencevich EN, Schwaber MJ, Karchmer AW, Carmeli Y.
Comparison of mortality associated with methicillin-resistant and methicillinsusceptible Staphylococcus aureus bacteremia: a meta-analysis. Clin. Infect. Dis. 36(1), 53-59 (2003).

7. Engemann JJ, Carmeli Y, Cosgrove SE et al. Adverse clinical and economic outcomes attributable to methicillin resistance among patients with Staphylococcus aureus surgical site infection. Clin. Infect. Dis. 36(5), 592-598 (2003).

8. Cosgrove SE, Qi Y, Kaye KS, Harbarth S, Karchmer AW, Carmeli Y. The impact of methicillin resistance in Staphylococcus aureus bacteremia on patient outcomes: mortality, length of stay, and hospital charges. Infect. Control Hosp. Epidemiol. 26(2), 166-174 (2005).

9. Shorr AF, Haque N, Taneja C et al. Clinical and economic outcomes for patients with health care-associated Staphylococcus aureus pneumonia. J. Clin. Microbiol. 48(9), 3258-3262 (2010).

10. Chambers HF, DeLeo FR. Waves of resistance: Staphylococcus aureus in the antibiotic era. Nat. Rev. Microbiol. 7(9), 629-641 (2009).
11. Hanberger $\mathrm{H}$, Walther S, Leone $\mathrm{M}$ et al. Increased mortality associated with metihcillin-resistant Staphylococcus aureus (MRSA) infection in the intensive care unit: results from the EPIC II study. Int. J. Antimicrob. Agents 38(4), 331-335 (2011).

12. Vincent JL, Rello J, Marshall J et al. International study of the prevalence and outcomes of infection in intensive care units. JAMA 302(21), 2323-2329 (2009).

- Large international study (EPIC II study), on which the Hanberger et al. [11] study is based.

13. Bootsma MC, Diekmann O, Bonten MJ. Controlling methicillin-resistant Staphylococcus aureus: quantifying the effects of interventions and rapid diagnostic testing. Proc. Natl Acad. Sci. USA 103(14), 5620-5625 (2006).

14. Jarlier V, Trystram D, Brun-Buisson C et al. Curbing methicillin-resistant Staphylococcus aureus in 38 French hospitals through a 15-year institutional control program. Arch. Intern. Med. 170(6), 552-559 (2010). 